\title{
Enhanced Indoor Location Tracking Through Body Shadowing Compensation
}

\author{
Jens Trogh*, David Plets*, Arno Thielens*, Luc Martens* and Wout Joseph* \\ * Department of Information Technology, iMinds - Ghent University, Belgium, jens.trogh@intec.ugent.be
}

\begin{abstract}
This paper presents a radio-frequency (RF) based location tracking system that improves its performance by eliminating the shadowing caused by the human body of the user being tracked. The presence of such a user will influence the RF signal paths between a body-worn node and the receiving nodes. This influence will vary with the user's location and orientation and, as a result, will deteriorate the performance regarding location tracking. By using multiple mobile nodes, placed on different parts of a human body, we exploit the fact that the combination of multiple measured signal strengths will show less variation caused by the user's body. Another method is to compensate explicitly for the influence of the body by using the user's orientation towards the fixed infrastructure nodes. Both approaches can be independently combined and reduce the influence caused by body shadowing, hereby improving the tracking accuracy. The overall system performance is extensively verified on a building-wide testbed for sensor experiments. The results show a significant improvement in tracking accuracy. The total improvement in mean accuracy is $38.1 \%$ when using three mobile nodes instead of one and simultaneously compensating for the user's orientation.
\end{abstract}

Index Terms-Tracking, Localization, Human Body Shadowing, Wireless Sensor Network, Indoor Environment

\section{INTRODUCTION}

Indoor localization and tracking systems have gained huge interest because of the many context-aware applications that have emerged lately. These applications are situated in domains such as the healthcare, the industry, and the cultural sector. Many localization systems already try to cope with performance deterioration caused by multipath fading and diffraction [1]. However, an important factor is the influence caused by the human body itself. The presence of a user's body can block the line-of-sight (LoS) between a body-worn node (or tag) and a receiving node, and cause additional propagation losses. These additional propagation losses are currently not accounted for and will generally decrease the accuracy of signal strength based localization systems. This effect has already been noted in literature but is often still neglected [2]. Many localization applications verify the performance of their developed system by stepwise moving a node placed on a tripod, hereby explicitly removing the human from the equation. However, practical human tracking applications, always imply the presence of a user's body. In this work, two novel approaches to mitigate the human body shadowing are investigated and combined in one location tracking system. First, placing multiple tags on different zones of the human body, which leads to received signal strength indicator (RSSI) values that show less variation caused by shadowing. This improves the tracking accuracy. A second strategy is to take into account the user's orientation towards the fixed infrastructure and the body-worn tag's relative position. A human body loss model can then be used to explicitly compensate for the user's influence. In this method, the orientation of a user is determined without making use of the classical compass or gyroscope approaches, but with a novel orientation estimator built on top of our tracking algorithm.

\section{RELATED WORK}

Current localization and tracking systems can be distinguished from each other in multiple ways [3]. Most localization methods use GPS [4], WiFi [5] or Ultra-wideband (UWB) [6] as information carrying signal. Another distinction can be made based on the used ranging technique, the most well known are: Angle of Arrival (AoA) [7], Angle of Departure (AoD) [8], Time (Difference) of Arrival (TDoA) [9] and Received Signal Strength Indication (RSSI) [5], [10], [11]. These ranging techniques are mostly used in combination with triangulation, trilateration and RSSI fingerprinting, respectively. Due to the complexity of many indoor environments multipath arises, which decreases the positioning accuracy. Current state-of-the-art tries to cope with this by making use of advanced processing techniques, e.g., Kalman filters [6], [12], particle filters [13]-[15] and machine learning techniques [16], but all aforementioned approaches neglect the influence of the human body itself. In [17], a body shadowing mitigation method is used on top of an RSSI-based Monte Carlo localization technique, achieving meter scale accuracies for a wrist-worn personnel tracking tag. The shadowing caused by a user's body is mitigated by using LoS and non-line-of-sight (NLoS) channel models. A disadvantage of this approach is that it depends on a manual differentiation of the LoS conditions and separate measurements that need to be conducted for each LoS condition. In [18], video cameras are used to detect the human orientation and an empirical compensation model is used to compensate for body electronic interference. In [19], multiple sensors are placed on a user and measured power level values are used for estimating the position and orientation of a user in a single room. A theoretical procedure to evaluate the maximum attainable performance with RSSI is presented. The analysis used in [19] is based on ray-tracing to compute a fine grid of RSSI values and a maximum-likelihood approach for localization. In [20], a fingerprinting system based on 
neural networks is used for indoor localization with Bluetooth devices. They use a compass module to provide information about the user's orientation, which improves the selection of the most adequate neural network to use. The achieved results are highly accurate, but a lot of training data is needed, since for every user orientation a neural network has to be trained. In [21], [22] the losses caused by a human body are used as an advantage. They ask the user to rotate in place, simulating the behavior of a directional antenna. This directional analysis technique is used to localize an outdoor access point (AP) in [21] and in [22] this is used to know your own location.

In our work, the user's direction is obtained with a novel orientation estimator, based on previous and current location predictions so the shadowing caused by the human body can be automatically mitigated. There is no need for manual differentiation [17], video cameras [18], or orientation tracking sensors like accelerometers, gyroscopes, and compasses [20]. The compensation model is based on three-dimensional electromagnetic simulations with a human phantom (no need for extensive measurements [17], [20]) and all possible orientations are taken into account. In this way, we obtain a continuous three-dimensional model to compensate for body shadowing. The performance evaluation is done at $2.4 \mathrm{GHz}$ using ZigBee nodes [23], on a building-wide testbed, not limited to a single room or theoretical framework [19].

\section{Motivation}

In this section, the influence of human body shadowing on the exchanged signals between a body-worn mobile device and a fixed AP, is investigated and characterized. This is relevant for all signal strength based localization and tracking algorithms which purpose is to track humans. In this work, we will verify the proposed body shadowing compensation methods with an RSSI fingerprinting based tracking algorithm [24]. Other signal strength based localization methods can also benefit from this approach, e.g.: the aforementioned RSSI based algorithms that use Kalman filter [12] or particle filters [13], [15]. Human body shadowing can also influence the performance of ToA based localization methods. The presence of a human body can e.g., block the line of sight path or cause the creation of additional paths with multipath, biases in the estimated delays and performance degradation as a result. The proposed methods in this work are aimed at signal strength based system, thus other methods will have to be developed but this is beyond the scope of the paper. In general, eliminating the influence of human shadowing results in better and more robust (ranging) measurements which, consequently, will lead to higher localization accuracies.

As mentioned before, human body shadowing occurs when the RF signal path between a body-worn tag and a receiving node is completely or partly blocked by a person. The exchanged RF signals are altered due to dielectric losses in the human body. This manifests itself as a drop in signal strength. Additional propagation losses of around 10 to $30 \mathrm{~dB}$ are reported in literature [21]. To verify this effect, an experiment is conducted: a user is asked to turn $360^{\circ}$ around its axis, while wearing a mobile tag on his chest and back (see Figure $4 \mathrm{a}$ and $4 \mathrm{~b}$ ). The user turns $45^{\circ}$ every 15 seconds, taking 2 minutes for a full rotation. The RSSI values from the packets sent by the mobile tags are measured by forty fixed APs (the blue dots in Figure 3, for more details on this testbed, see Section V). In Figure 1, the measured RSSI values as function of the user's orientation are shown for a nearby $(12 \mathrm{~m})$ and far away $(66 \mathrm{~m})$ AP. The location of the user and APs are indicated with a yellow circle in Figure 3. An averaging window of 15 seconds is used.

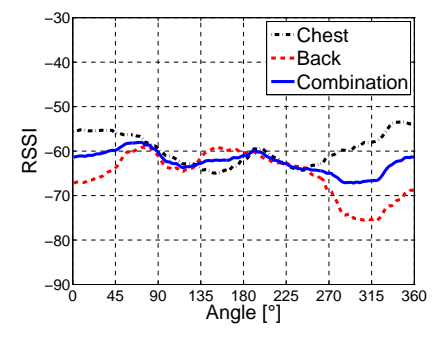

(a) Nearby AP (12 m)

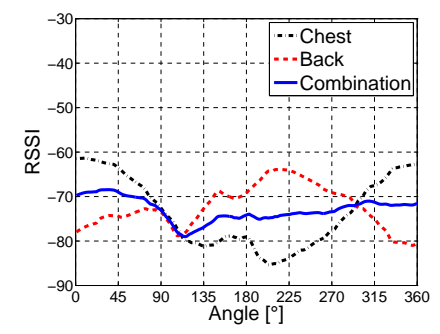

(b) Far away AP (66 m)
Figure 1: Human body shadowing while rotating a human body in an indoor environment.

From Figure 1, it is clear that the orientation of a user has an influence on the measured RSSI values (on top of the variance caused by multipath). A high RSSI value measured by the chest tag corresponds always to a low RSSI value measured by the back tag (and the other way around). This is because the human body is located between both bodyworn tags and can e.g. block or attenuate the strongest path between the APs and mobile tags. The maximum observed differences between the chest and back tag, measured by the forty fixed APs, range from $1.8 \mathrm{~dB}$ to $25.2 \mathrm{~dB}$ with an average value of $14.8 \mathrm{~dB}$. The highest differences are observed in LoS situations, whereas the lowest differences are observed in situations where the RF signal suffers from severe multipath. As a result, the average RSSI value of both tags shows less variation. To be more specific, the standard deviation, averaged over all forty APs, is $4.9 \mathrm{~dB}, 4.3 \mathrm{~dB}$, and $2.5 \mathrm{~dB}$ for the chest, back, and combination of both tags, respectively. This means that the orientation of a user will have less influence when multiple mobile tags are combined.

\section{OVERVIEW OF THE SYSTEM}

In this section, a complete overview of the location tracking system is given. First, the tracking algorithm that serves as a basis for this work is explained. Then, the impact of the human body on the received signal strength is shown. Next, the two solutions to mitigate this body shadowing effect are presented. In the first solution, it is investigated how this effect can be reduced by using multiple body-worn tags. In the second solution, it is described how the tracking accuracy can be further improved, by incorporating the user's orientation and using a compensation model. Finally, the different compensation 
models are discussed. A flow graph of the complete location tracking system can be found in Figure 2.

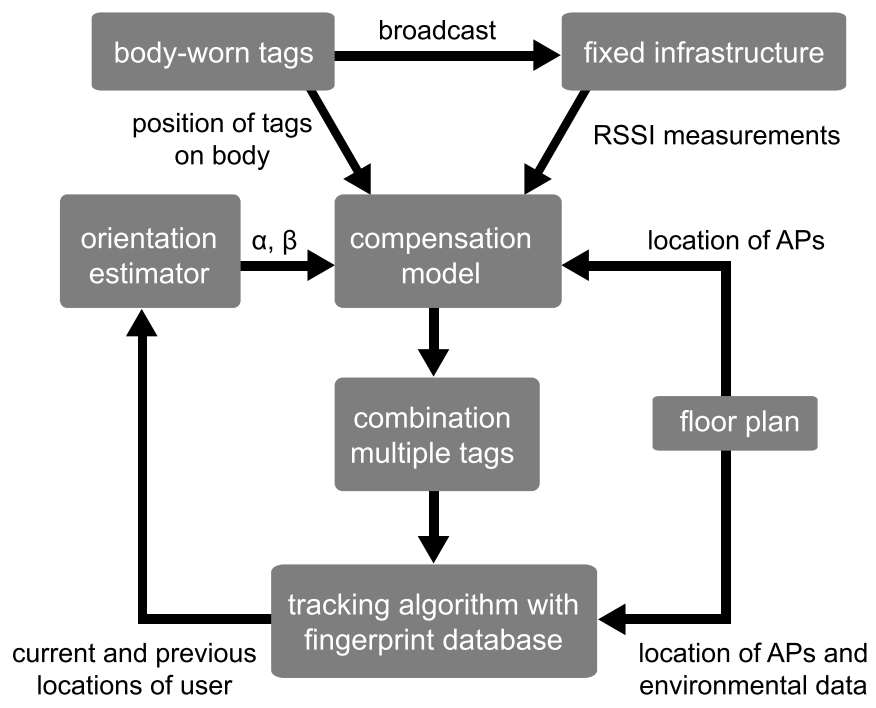

Figure 2: Flow graph of the complete location tracking system.

The body-worn tags broadcast packets that are received by the APs (fixed infrastructure). The measured RSSI values and positions of the tags on the user's body are passed to the compensation model. This model uses the angles from the orientation estimator and the locations of the APs from the floor plan (Figure 3), to determine the influence caused by the user's body (Section IV-C2). The compensated RSSI values are firstly passed to the combination of multiple tags block (Section IV-B) and finally, to the tracking algorithm (Section IV-A). This algorithm uses a fingerprint database and a floor plan (for environmental data and the locations of the APs), as additional inputs. The predicted current and previous locations are fed back to the orientation estimator to estimate the next orientation (Section IV-C1).

\section{A. Tracking algorithm}

A Viterbi-like tracking algorithm that uses off-the-shelf devices and the well-known RSSI fingerprinting technique serves as starting point for this work [24]. In the standard RSSI fingerprinting technique (without the Viterbi principle) the current location is estimated by comparing the RSSI measurements with reference values for a certain position from a fingerprinting database, which results in the following cost function:

$$
\operatorname{cost}^{\text {simple }}=\sum_{n=1}^{N}\left|R S S I_{n}^{\text {meas }}-R S S I_{n}^{\text {ref }}\right|
$$

cost $t^{\text {simple }}$ is the cost function of the RSSI fingerprinting technique, which is referred to as simple. $N$ is the number of APs that measure the RSSI values from the packets broadcasted by the body-worn $\operatorname{tag}(\mathrm{s}) . R S S I_{n}^{\text {meas }}$ is the measured RSSI values from AP $n$. $R S S I_{n}^{\text {ref }}$ is the reference RSSI value from AP $n$ for a certain position and the position corresponding with the lowest cost is taken as most likely current location. The more advanced Viterbi-like tracking algorithm uses the environment of the user that is being tracked and a motion model as constraints to determine the most likely sequence of positions (path) instead of only the most likely current position (Viterbi principle). These two constraints ensure that no walls are crossed and that no unrealistically large distances are traveled within a given time frame. The following cost function is used to determine the most likely path (after applying both constraints):

$$
\operatorname{cost}_{i, T}^{\text {advanced }}=\sum_{t=1}^{T} \sum_{n=1}^{N}\left|R S S I_{t, n}^{\text {meas }}-R S S I_{t, n, i}^{r e f}\right|
$$

cost $_{i, T}^{\text {advanced }}$ is the associated cost of the $i$ th path stored in memory after $T$ time steps. $R S S I_{t, n}^{\text {meas }}$ is the RSSI measurement at time step $t$ from $\operatorname{AP} n . R S S I_{t, n, i}^{r e f}$ is the reference RSSI value from AP $n$ for the position along path $i$ at time step $t$ (note that the reference RSSI values for a certain position are static, the subscript $t$ is only used to indicate the position along path $i$ ). The last position of the path with the lowest associated cost is taken as most likely current location. The calculations of paths and costs are not restarted every time a new measurement is received but the paths and costs from a previous iteration serve as input for the current iteration along with the new measurements. The reference RSSI values are stored in a fingerprint database and are derived from a path loss (PL) value calculated with a theoretical model from a network planner (WHIPP tool [25]):

$$
P L_{r e f}=\underbrace{P L_{0}+10 \gamma \log _{10}\left(\frac{d}{d_{0}}\right)}_{\text {distance loss }}+\underbrace{\sum_{i} L_{W_{i}}}_{\text {cumulated wall loss }}+\underbrace{\sum_{j} L_{B_{j}}}_{\text {interaction loss }}
$$

$P L_{\text {ref }}[\mathrm{dB}]$ is the total path loss calculated with the theoretical model, $P L_{0}[\mathrm{~dB}]$ is the path loss at a reference distance $d_{0}[\mathrm{~m}], \gamma[-]$ is the path loss exponent and $d[\mathrm{~m}]$ is the distance along the path between transmitter and receiver. The first two terms represent the path loss due to the traveled distance (distance loss), the third term (cumulated wall loss) is the sum of all wall losses $L_{W_{i}}$ when a signal propagates through a wall $W_{i}$, and the fourth term (interaction loss) takes into account the cumulated losses $L_{B_{j}}$ caused by all propagation direction changes $B_{j}$ of the propagation path from transmitter to receiver. The only prerequisite to generate this fingerprint database for a certain building is to draw its floor plan with the right materials in the WHIPP tool (no additional measurements are needed). Most common materials are already available in the tool: brick, drywall, wood, glass and metal, both in thin and thick format. The material parameters come from literature. The tool itself was verified in three other types of buildings: a retirement home, a congress center, and an arts center. Without any additional measurements or tuning, the predictions were also excellent. The fingerprint database has a density of $50 \mathrm{~cm}$ in all performance evaluations. In [24] it was proven that a higher density would not further improve the results and that a density of $50 \mathrm{~cm}$ is suited to work in real time. Using a theoretical model avoids an expensive 


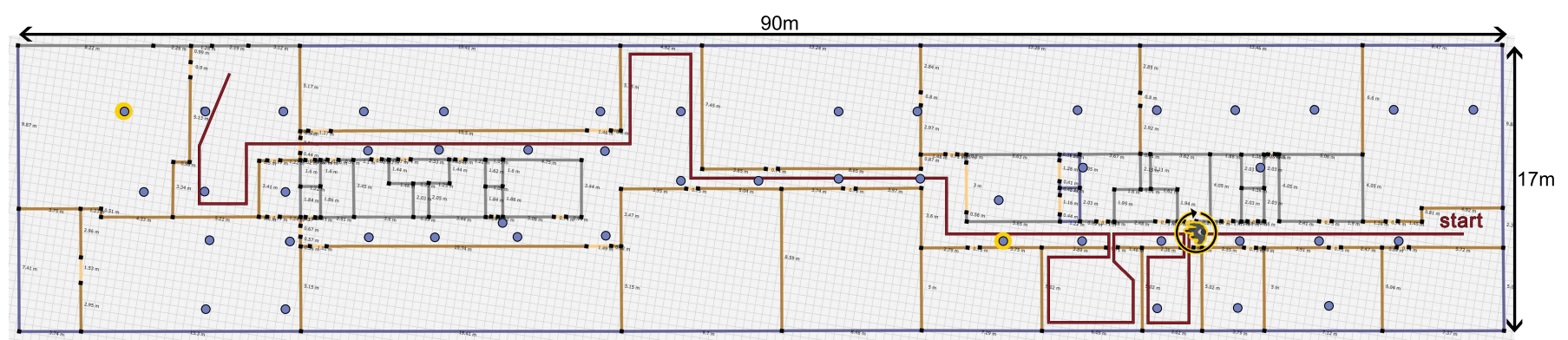

Figure 3: Testbed floor plan with test trajectory (red) and locations of APs (blue) and rotating user (yellow).

and time consuming measurement campaign but allows for an immediate deployment at the expense of a slightly reduced accuracy.

\section{B. Solution 1: combining multiple tags}

As indicated in Section III, the measurements of multiple mobile tags worn on different parts of the human body can be combined to reduce the influence of body shadowing. This results in a closer match between the preprocessed measurements and the reference RSSI values from the fingerprinting database, which suggests a possible improvement in tracking accuracy. Determining the optimal number and optimal positions for the mobile tags depends on the required accuracy and wearing comfort in particular. Our goal is to improve the performance while maintaining practical tag positions. Three positions are considered: the central area of the chest and back (to have diversity in the forward and backward direction) and the right wrist (can be worn like or integrated in a watch, see Figure 4).

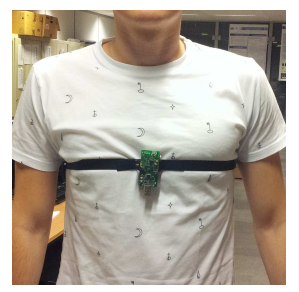

(a) Chest

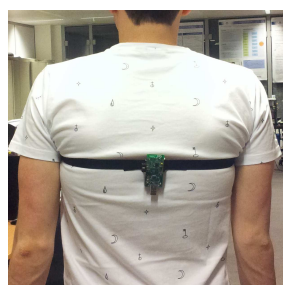

(b) Back

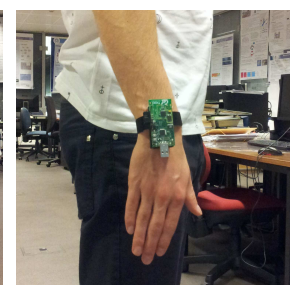

(c) Wrist
Figure 4: Body-worn tags.

These body-worn tags broadcast packets and the measured RSSI values are combined in following cost function (based on Equation 2):

$$
\operatorname{cost}_{i, T}^{\text {combine }}=\sum_{t=1}^{T} \sum_{n=1}^{N} \sum_{p=1}^{P}\left|R S S I_{t, n, p}^{\text {meas }}-R S S I_{t, n, i}^{\text {ref }}\right|
$$

$R S S I_{t, n, p}^{\text {meas }}$ is the RSSI measurement at time step $t$ from AP $n$ of a packet broadcasted by body-worn tag $p . P$ is the amount of mobile tags that are in use and $p$ is a number referring to the body-worn tag's position, i.e., 1 = chest, 2 = back, and $3=$ wrist.

\section{Solution 2: compensating for user's orientation}

The second solution compensates explicitly for the influence caused by a user with a certain position and tag orientation towards the infrastructure APs. It consists of two parts: an orientation estimator and a compensation model.

1) Orientation estimator: Due to the nature of most building structures, people tend to walk in the same direction for at least a few seconds. This behavior can be exploited to estimate a user's orientation. More specifically, the angle between previous and current location predictions can be used as an estimation for the next orientation. Such orientation estimator can be built on top of an existing tracking system which provides the current and previous positions as input. The next orientation is predicted as:

$$
\begin{gathered}
O_{t+1}^{e s t}=\frac{180}{\pi} \arctan \left(\frac{P_{t, y}-P_{t, y}^{a m}}{P_{t, x}-P_{t, x}^{a m}}\right) \\
P_{t}^{a m}=\frac{1}{K} \sum_{k=1}^{K} P_{t-k}
\end{gathered}
$$

$O_{t+1}^{e s t}\left[^{\circ}\right]$ is the estimated next orientation, $P_{t}^{a m}$ is the arithmetic mean of $K$ previous predicted positions $P_{t}$ at time step $t$ and the $x$ and $y$ subscript indicate the $\mathrm{x}$ and y coordinates, respectively. An arctangent function with two arguments (atan2) is used to obtain the appropriate quadrant of the computed angle. The performance of this orientation estimator will depend on the accuracy of the tracking system itself and on the movement of a user (i.e., frequency of turns taken and standing still moments). The goal is to investigate whether human body shadowing can be mitigated with this approach, instead of using the more precise measurements of an accelerometer, compass, or gyroscope (at the cost of additional required hardware). For now, it is assumed that the user is walking forward. The robustness and responsiveness of the estimator are two contradictory demands. Including more previous location predictions (parameter $K$ ) will improve the performance of the estimator when few turns are present but this will rapidly decrease when a trajectory with more abrupt changes, is followed. The reason for the improvement is that the predicted locations are never completely accurate (e.g., variations around an actually followed straight line) and taking into account multiple positions can reduce the effect 
of prediction inaccuracies (averaging out the error). Because a user will not keep walking along this straight line, the improvement will stop and the orientation error will start to deteriorate when even more previous positions are taken into account. A simulation was performed to evaluate our estimator: two trajectories were outlined on a floor plan. The first trajectory has a length of $100 \mathrm{~m}$ and contains twenty-three $90^{\circ}$ turns and two $180^{\circ}$ turns, which results in an average of one turn every $4 \mathrm{~m}$ (blue trajectory in Figure 5). The second one is a more straight trajectory, it has a length of $56 \mathrm{~m}$ and contains eight $90^{\circ}$ turns, which results in an average of one turn every $7 \mathrm{~m}$ (red trajectory in Figure 5). The walking speed was set to $1 \mathrm{~m} / \mathrm{s}$.

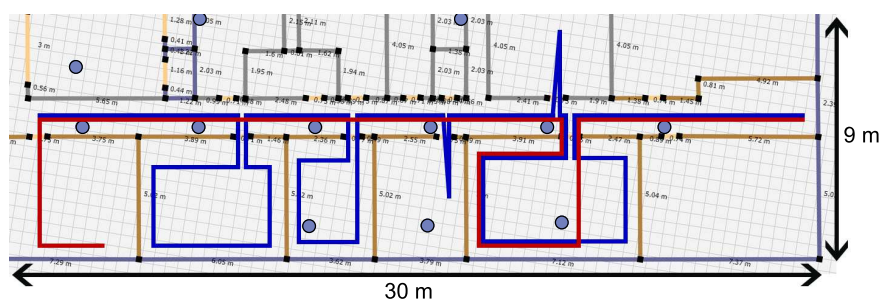

Figure 5: Blue and red trajectory for testing the orientation estimator (simulation).

The RSSI values corresponding to the positions along the trajectory were picked from the fingerprint database from Section IV-A and are used as input for the tracking algorithm. Gaussian white noise with standard deviations of $1 \mathrm{~dB}, 3 \mathrm{~dB}$, and $6 \mathrm{~dB}$ was added to these RSSI values to simulate more realistic conditions. Finally, the positions predicted by the tracking algorithm are used to estimate the orientation with equations 5 and 6 . The user's orientation ranges from $0^{\circ}$ to $360^{\circ}$, meaning that an orientation error of $180^{\circ}$ is the worst possible result. The orientation error is defined as:

$$
\epsilon_{\text {or }}= \begin{cases}\left|O^{\text {est }}-O^{\text {exact }}\right| & \left|O^{\text {est }}-O^{\text {exact }}\right| \leq 180^{\circ} \\ 360^{\circ}-\left|O^{\text {est }}-O^{\text {exact }}\right| & \left|O^{\text {est }}-O^{\text {exact }}\right|>180^{\circ}\end{cases}
$$

$\epsilon_{o r}$ is the orientation error, $O^{e s t}$ is the estimated orientation and $O^{\text {exact }}$ is the exact orientation, which can be easily derived from the trajectories of Figure 5 (these trajectories are known in advance). Figure 6 shows a plot of the median orientation errors as a function of the number of previous locations taken into account (parameter $K$ from equation 6). The simulations were repeated five times for averaging purposes.

As expected, the orientation error will first decrease when more previous positions are taken into account (averaging out inaccuracies) and will then increase again (due to turns taken). For the blue trajectory (with many turns), the optimal value for $K$ is 3 with orientation errors of $12^{\circ}, 22^{\circ}$, and $32^{\circ}$, depending on the amount of added noise. For the red trajectory, this optimal value for $K$ is 5 with orientation errors of $10^{\circ}$, $15^{\circ}$, and $22^{\circ}$, depending on amount of added noise. Because the followed trajectory is unknown beforehand, the default $K$ value is set to 4 , which can deal with more abrupt trajectory changes and is still sufficiently accurate to compensate for the

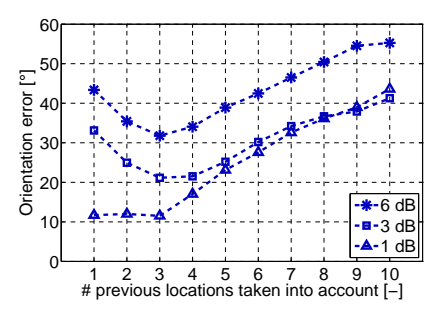

(a) Blue trajectory

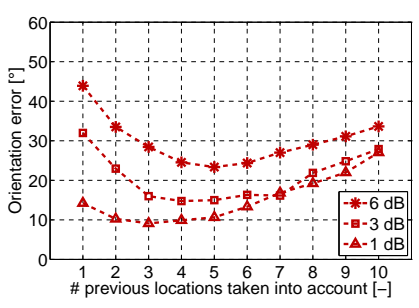

(b) Red trajectory
Figure 6: Performance of orientation estimator for the two trajectories and three noise levels.

user's orientation (see Section VI-B). The biggest advantage of this approach is the ease of use and that there is no need for additional hardware to obtain the user's orientation. Disadvantages are the inability to detect a user rotating in place and the rather harsh orientation estimations. In Section VI-B it is shown that this approach suffices to reduce the impact of human body shadowing.

Once the orientation of a user is known, the azimuth angle $\alpha$ and the elevation angle $\beta$ between the body-worn $\operatorname{tag}(\mathrm{s})$ and the fixed infrastructure APs can be calculated (see Figure 7). It is assumed that the tag's position on the body and a floor plan with the locations of the fixed APs are known beforehand and that the user is walking forward.

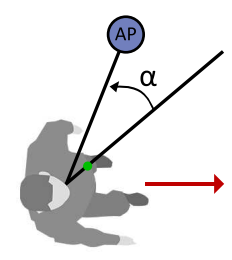

(a) Top view

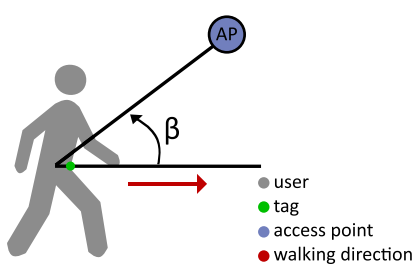

(b) Side view
Figure 7: Angles between body-worn tag and AP.

2) Compensation models: Next, a compensation model is needed to estimate the influence due to the presence of the user. The mobile tags are calibrated based on the same measurements from Section III: the average value between the measurements and the RSSI fingerprinting database is taken as offset. This offset is inherent to the mobile tags and their transmit power, and is calculated once. This means that the measured RSSI values will be an overestimation when tag and receiving node face each other directly and be an underestimation when the human body is completely blocking the signal (see Figure 1). Two compensation models were used: a basic over/underestimation model and a simulation based three-dimensional model:

- The first model labels the measurements as over- or underestimated values based on the azimuth angle $\alpha$ between tag and receiving node:

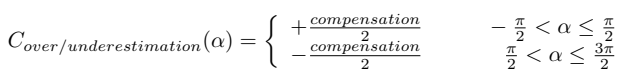


This over/underestimation compensation model is plotted in Figure 9a. The default value of the compensation parameter from Equation 8 is set to $6 \mathrm{~dB}$, which was found to be a good compromise between compensating for worst case body shadowing and when almost no shadowing is present. (The maximum observed differences between the chest and back tag, measured by the forty fixed APs, varied from $1.8 \mathrm{~dB}$ to $25.2 \mathrm{~dB}$, see Section III.)

- The second model is based on simulations carried out in Semcad X, a three-dimensional full wave simulation environment based on the finite-difference timedomain (FDTD) method. The simulations are executed at $2.45 \mathrm{GHz}$ using the Virtual Family Male (VFM) [26]. The VFM is a heterogeneous phantom with a $\mathrm{BMI}=22.3 \mathrm{~kg} / \mathrm{m}^{2}$. An accurate model of the integrated onboard antenna of our mobile tag was built in the simulation platform [27] (see Figure 8a). This Planar Inverted-F antenna (PIFA) was made out of metal and mounted on a $1 \mathrm{~mm}$ thick, dielectric substrate with a relative permittivity of 4 and measures $65 \mathrm{~mm}$ by $31 \mathrm{~mm}$ [23]. The antenna is optimized to resonate at $2.45 \mathrm{GHz}$ (a plot of the reflection coefficient S11 [dB] can be found in Figure $8 b$ ).

The mobile tag was placed on various parts of the VFM: chest, back, and right wrist. The simulated directivity pattern reflects the influence of the human body (see Figure 8c). The values of the directivity pattern are shifted in such a way that the average value is zero and the range is normalized to the same compensation value of Equation 8. In this way a three-dimensional compensation model, that reflects the influence caused by the human body, is obtained. The second model is expressed as $C_{\text {semcad,p }}(\alpha, \beta)$ ( $\alpha$ and $\beta$ are the azimuth and elevation angle between tag and receiving node, and $p$ indicates the tag position).

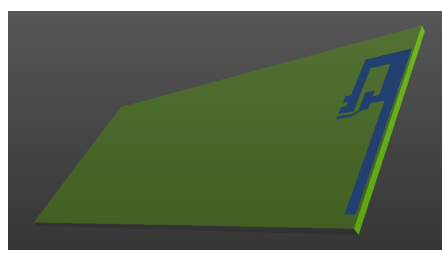

(a) Substrate with PIFA

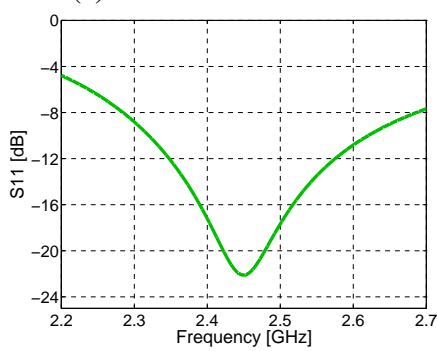

(b) Reflection coefficient S11

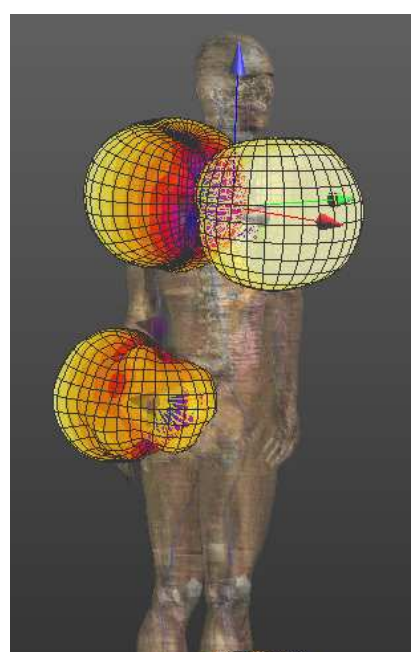

(c) Directivity patterns
Figure 8: Simulations in Semcad X.

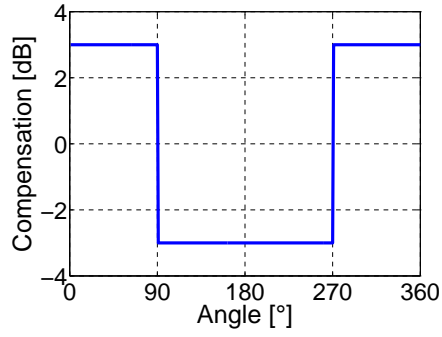

(a) over/underestimation

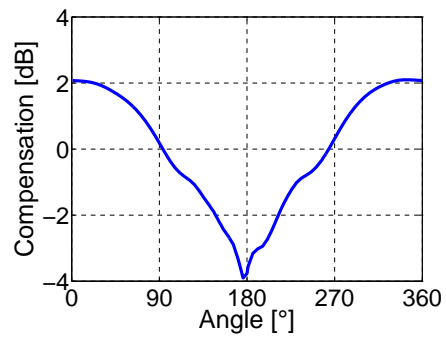

(c) semcad: back

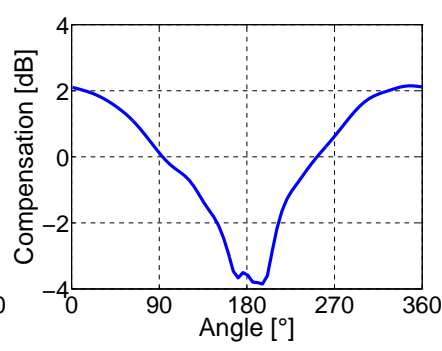

(b) semcad: chest

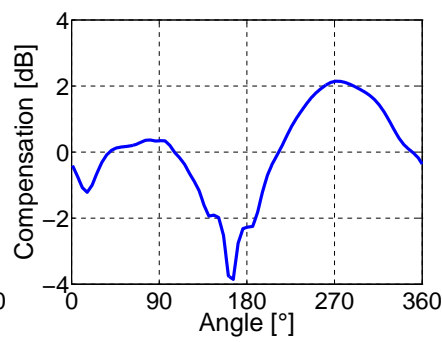

(d) semcad: wrist
Figure 9: Graphical representation of the two compensation models.

Including the compensation for a user's orientation, results in following cost function for the tracking algorithm (based on Equation 4):

$$
\operatorname{cost}_{i, T}^{\text {compensate }}=\sum_{t=1}^{T} \sum_{n=1}^{N} \sum_{p=1}^{P}\left|\left(R S S I_{t, n, p}^{\text {meas }}-C_{j, p}(\alpha, \beta)\right)-\operatorname{RSSI}_{t, n, i}^{\text {ref }}\right|
$$

$C_{j, p}(\alpha, \beta)$ is the $j$ th compensation model, for mobile tag $p$ and uses the angles $\alpha$ and $\beta$ as input arguments (the subscript $j$ is either over/underestimation or semcad). The other symbols are already defined in Sections IV-A and IV-B. Note that the over/underestimation compensation model makes no use of the elevation angle $\beta$ and uses the same model for all mobile tags $p$. In Figure 9, a graphical representation of the two compensation models is shown. To obtain a two-dimensional plot, an elevation angle $\beta$ of $20^{\circ}$ was chosen for the semcad compensation model.

It is clear from Figure 9 that the semcad compensation models do depend on the tag position but there are many similarities, which can be expected because the source of influence is the same user. Only the compensation model for a wrist-worn tag clearly deviates from the others, due to the used human posture where there is some open space between arm and body.

\section{EXPERIMENT CONFIGURATION}

The experiments are conducted on a wireless testbed, located on the third floor of an office building in Ghent, covering over $1500 \mathrm{~m}^{2}(17 \mathrm{~m}$ by $90 \mathrm{~m}$, see Figure 3$)$. It consists of several computer labs, offices, and meeting rooms. The core is made of concrete walls, the movable inner structure is made of layered drywall and the doors are made of wood. The wireless network consists of 48 fixed sensor nodes that 
were installed at a height of $2.5 \mathrm{~m}$ (blue dots in Figure 3). TelosB motes from Crossbow are used as the body-worn tags [23]. These are equipped with an embedded PIFA antenna and Chipcon CC2420 radio operating at $2.4 \mathrm{GHz}$ (IEEE 802.15.4/ZigBee compliant [28]). There are 31 transmission power levels between -25 and $0 \mathrm{dBm}$ (set to $0 \mathrm{dBm}$ in all experiments). The mobile tags broadcast 10 packets per second which are received by the infrastructure nodes. Every second a location update is generated (the average RSSI values of the packets received within this second are used as input for the tracking algorithm). The test trajectory (indicated in red in Figure 3) has a total length of $140 \mathrm{~m}$, passes through three meeting rooms, a computer lab, and the hallway (note that there are no APs installed in the second meeting room). The ground truth, i.e., the correct locations for comparison, are provided by fragmenting the test trajectory based on the number of location updates from an experiment (the user walked as continuously as possible with an average speed of $1.2 \mathrm{~m} / \mathrm{s})$.

\section{PERformance EVAluation}

This section investigates the impact of using multiple mobile tags and body shadowing compensation on the tracking accuracy. The presented results are obtained from experiments performed on the testbed described in Section $\mathrm{V}$. The mean, standard deviation, 50th and 95th percentile value of the tracking accuracy are used as evaluation metrics. This accuracy is defined as the Euclidean distance between the predicted and actual location:

$$
\text { accuracy }=\sqrt{\left(x_{\text {pred }}-x_{a c t}\right)^{2}+\left(y_{\text {pred }}-y_{a c t}\right)^{2}}
$$

The predicted and actual position are located at coordinates $\left(x_{\text {pred }}, y_{\text {pred }}\right)$ and $\left(x_{a c t}, y_{\text {act }}\right)$, respectively.

\section{A. Impact of multiple mobile tags}

To evaluate the performance when using multiple tags, the test trajectory was repeated 5 times by a human wearing three tags. The tags were placed on the central area of the chest, back, and right wrist (see Figure 4). The two localization algorithms from Section IV-A are used for testing. The standard RSSI fingerprinting technique is referred to as simple and the Viterbi-based tracking algorithm is referred to as advanced. The measured RSSI values from the mobile tags are combined with the cost function from Section IV-B. Figure 10 shows the mean tracking accuracy along with the standard deviations as error bars. These accuracies are always averaged over the mobile tags, i.e., the results with one tag are the average of the three tags separately, the results with two tags are the average of the three possible combinations of two tags (chest + back, chest + wrist , and back + wrist) and for the results with three tags there is only one combination (chest + back + wrist $)$.

Figure 10 shows that the mean tracking accuracy and the standard deviation improve as more mobile tags are used, for both localization algorithms. The relative improvement in

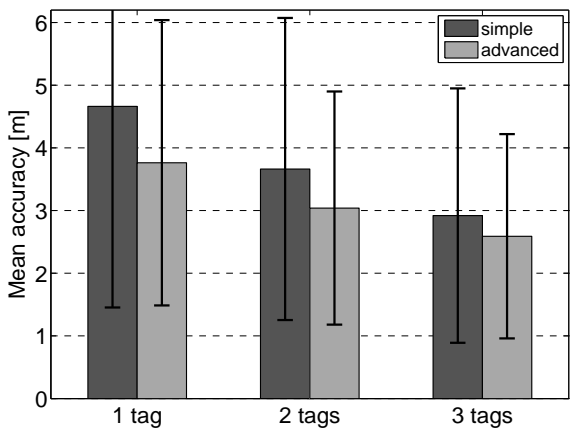

Figure 10: Mean accuracy and standard deviation as a function of the number of used mobile tags for a simple and advanced localization algorithm.

mean accuracy is $19.2 \%$ (from $3.76 \mathrm{~m}$ to $3.04 \mathrm{~m}$ ) when using two instead of one mobile tag, $14.8 \%$ (from $3.04 \mathrm{~m}$ to $2.59 \mathrm{~m}$ ) when using three instead of two mobile tags, and $31.2 \%$ (from $3.76 \mathrm{~m}$ to $2.59 \mathrm{~m}$ ) when using three instead of one mobile tag (calculated for advanced from Figure 10). The improvements in standard deviation (plotted as error bars in Figure 10) are similar: $18.3 \%$ (from $2.28 \mathrm{~m}$ to $1.86 \mathrm{~m}$ ), $12.4 \%$ (from $1.86 \mathrm{~m}$ to $1.63 \mathrm{~m}$ ), and $28.4 \%$ (from $2.28 \mathrm{~m}$ to $1.63 \mathrm{~m}$ ), respectively. The 50th and 95th percentile value of the localization accuracy and the improvement of the advanced compared to the simple algorithm are summarized in Table I.

Table I: 50th and 95th percentile value of the accuracy for the simple and advanced localization algorithm.

\begin{tabular}{c|c|c|c|c|c|c}
\hline \hline Algorithm $\rightarrow$ & \multicolumn{2}{|c|}{ Simple } & \multicolumn{2}{c|}{ Advanced } & \multicolumn{2}{c}{ Improvement } \\
\hline$\#$ tags $\downarrow$ & 50 th [m] & 95 th [m] & 50th [m] & 95th [m] & 50th [\%] & 95th [\%] \\
\hline 1 & 4.17 & 10.68 & 3.48 & 8.50 & 16.6 & 20.5 \\
2 & 3.28 & 7.89 & 2.77 & 6.71 & 15.5 & 15.0 \\
3 & 2.63 & 6.41 & 2.30 & 5.66 & 12.6 & 11.7
\end{tabular}

The 50th and 95th percentile value improve in a similar way as the mean accuracy, when more mobile tags are used. Furthermore, the advanced algorithm always outperforms the simple one, as was already shown in [24]. More specific, the 50th percentile value improves by $16.6 \%, 15.5 \%$, and $12.6 \%$ when using one, two, and three mobile tags, respectively. The 95th percentile improvements are similar.

\section{B. Impact of body shadowing compensation}

The orientation estimator and three-dimensional compensation model (semcad) are verified with the same measurement data from Section VI-A. The Viterbi-based tracking algorithm (advanced) is used to provide the previous positions that are needed to estimate the user's orientation (see Section IV-C1). Three scenarios are considered: no compensation, compensation with the estimated orientation, and compensation with the exact orientation. The first scenario is the same as the best result from Section VI-A (advanced in Figure 10). The second and third scenario compensate for the influence of a user's body with the three-dimensional semcad compensation model from Section IV-C2. The only difference between them is that 
the second scenario uses the orientation estimator to determine the user's orientation, whereas the third scenario gets the exact orientation as an additional input. This is possible because the followed trajectory is known beforehand and it is assumed a user walks forward, hence the exact orientation can be determined. The third scenario can be considered as a reference scenario.

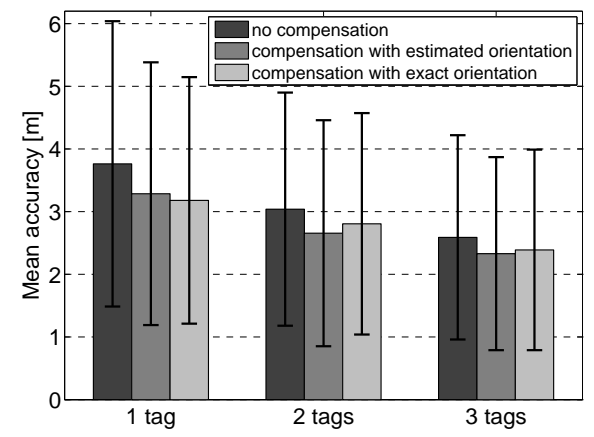

Figure 11: Impact of body shadowing compensation on the tracking accuracy.

From Figure 11 it is clear that including the user's orientation to compensate for human body shadowing will always improve the tracking accuracy (on top of the improvement due to using multiple mobile tags). Usage of our orientation estimator and compensation model result in additional improvements of $12.7 \%$ (from $3.76 \mathrm{~m}$ to $3.29 \mathrm{~m}$ ), $12.6 \%$ (from $3.04 \mathrm{~m}$ to $2.66 \mathrm{~m}$ ), and $10.0 \%$ (from $2.59 \mathrm{~m}$ to $2.33 \mathrm{~m}$ ) in mean accuracy for the situations with one, two, and three mobile tags, respectively. The improvements in standard deviation (error bars in Figure 11) are: $7.9 \%$ (from $2.28 \mathrm{~m}$ to $2.10 \mathrm{~m}$ ), $3.0 \%$ (from $1.86 \mathrm{~m}$ to $1.80 \mathrm{~m}$ ), and $5.5 \%$ (from $1.63 \mathrm{~m}$ to $1.54 \mathrm{~m})$, respectively. The total improvement in mean accuracy when using three mobile tags and compensating for body shadowing, compared to only one mobile tag and no compensation, is $38.1 \%$ (from $3.76 \mathrm{~m}$ to $2.33 \mathrm{~m}$ ). The 50th and 95th percentile value of the localization accuracy and the improvement of the compensation method compared to no compensation are summarized in Table II.

Table II: 50th and 95th percentile value of the accuracy with and without compensation.

\begin{tabular}{c|c|c|c|c|c|c}
\hline \hline & \multicolumn{2}{|c|}{ No compensation } & \multicolumn{2}{c|}{ Compensation } & \multicolumn{2}{c}{ Improvement } \\
\hline \# tags $\downarrow$ & 50 th $[\mathrm{m}]$ & 95 th $[\mathrm{m}]$ & 50 th $[\mathrm{m}]$ & 95 th $[\mathrm{m}]$ & 50 th $[\%]$ & 95 th [\%] \\
\hline 1 & 3.48 & 8.50 & 2.99 & 7.56 & 14.1 & 11.0 \\
2 & 2.77 & 6.71 & 2.28 & 6.52 & 17.7 & 2.8 \\
3 & 2.30 & 5.66 & 1.91 & 5.23 & 17.0 & 7.6
\end{tabular}

The median accuracy (50th percentile value) is now below $2 \mathrm{~m}$ when using all three tags and the body shadowing compensation. The improvements in median accuracy are somewhat higher compared to the ones in mean accuracy.

The median orientation error for this trajectory (averaged over the 5 runs) was $39^{\circ}, 35^{\circ}$, and $28^{\circ}$, for one, two, and three tags, respectively (which is comparable to the simulations from Section IV-C1). Furthermore, using the exact orientation has no added value compared to using the developed orientation estimator. Normally, this exact orientation is provided by a compass or gyroscope, but here this is accounted for by giving the exact orientation as an additional input. Therefore, using a compass or gyroscope has no significant added value for body shadowing compensation. In other localization schemes they can be used as an additional feature to estimate the position itself, for example in dead reckoning their usage will be beneficial [29].

\section{Comparison of compensation models}

The compensation with exact orientation from Section VI-B is used to evaluate the compensation models. In this way it is assured that the orientation estimator (and indirectly the tracking algorithm itself) has no impact on the comparison.

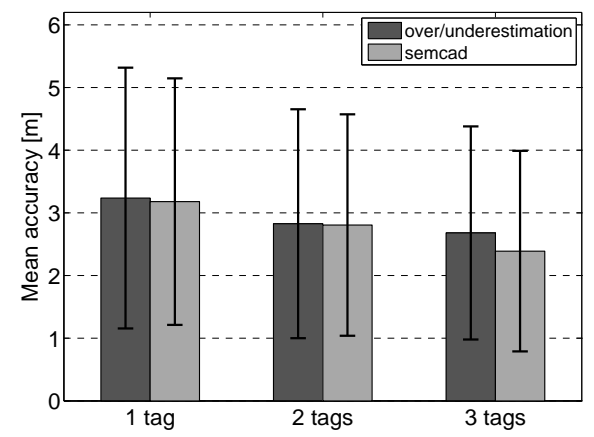

Figure 12: Comparison of different compensation models on the tracking accuracy.

Figure 12 shows the comparison between the over/underestimation and semcad compensation models from Section IV-C2. The three-dimensional model (semcad) still has the most accurate results for all tag combinations, but the benefit is limited. Only for three tags it performs clearly better: an improvement of $10.8 \%$ in mean accuracy (and $5.9 \%$ in standard deviation).

Although, simulations to determine the directivity pattern only have to be performed once (per position and mobile tag), they are rather time consuming. It will depend on the application if it is worth performing these to obtain more detailed models.

\section{CONCLUSIONS}

In this paper, novel techniques to reduce the effects of body shadowing on a tracking algorithm's performance, are presented. Two methods were found to be effective. The first one combines multiple mobile tags, placed on different parts of the human body. The second one compensates explicitly for the body shadowing caused by the user that is being tracked. Both methods can be independently combined, resulting in the most accurate performance. The first method exploits the fact that measurements performed by these multiple body-worn tags will show less variation (caused by the user's body) when combined. The second method uses a compensation model based on three-dimensional electromagnetic simulations with 
a human phantom and relies on the orientation of a user's tag towards the infrastructure nodes. The user's orientation is provided by an estimator developed on top of a tracking algorithm. This alleviates the need for specialized hardware like a compass or gyroscope and achieves similar performance. Using three instead of one body-worn tag results in a mean accuracy of $2.59 \mathrm{~m}$ (an improvement of $31.2 \%$ ). Compensating for the user's orientation, further improves this result to a mean accuracy of $2.33 \mathrm{~m}$ (a total and additional improvement of $38.1 \%$ and $10.0 \%$, respectively). Future work will include methods to obtain location-dependent compensation models by making use of environmental and empirical data. Furthermore, it will be explored whether compensating for the human body shadowing can improve other localization techniques.

\section{REFERENCES}

[1] Paramvir Bahl and Venkata N Padmanabhan. Radar: An in-building rfbased user location and tracking system. In INFOCOM 2000. Nineteenth Annual Joint Conference of the IEEE Computer and Communications Societies. Proceedings. IEEE, volume 2, pages 775-784. Ieee, 2000.

[2] Paramvir Bahl, Venkata N Padmanabhan, and Anand Balachandran. Enhancements to the radar user location and tracking system. Technical report, technical report, Microsoft Research, 2000.

[3] Long Cheng, Chengdong Wu, Yunzhou Zhang, Hao Wu, Mengxin Li, and Carsten Maple. A survey of localization in wireless sensor network. International Journal of Distributed Sensor Networks, 2012, 2012.

[4] P Misra and P Enge. Special issue on global positioning system. Proceedings of the IEEE, 87(1):3-15, 1999.

[5] Moustafa Youssef and Ashok Agrawala. The horus wlan location determination system. In Proceedings of the 3rd international conference on Mobile systems, applications, and services, pages 205-218. ACM, 2005.

[6] Paul Meissner, Erik Leitinger, Markus Fröhle, and Klaus Witrisal. Accurate and robust indoor localization systems using ultra-wideband signals. arXiv preprint arXiv:1304.7928, 2013.

[7] Eiman Elnahrawy, John Austen-Francisco, and Richard P Martin. Adding angle of arrival modality to basic rss location management techniques. In Wireless Pervasive Computing, 2007. ISWPC'07. 2nd International Symposium on. IEEE, 2007.

[8] Xinning Wei, Nico Palleit, and Tobias Weber. Aod/aoa/toa-based 3d positioning in nlos multipath environments. In Personal Indoor and Mobile Radio Communications (PIMRC), 2011 IEEE 22nd International Symposium on, pages 1289-1293. IEEE, 2011.

[9] Davide Dardari, Andrea Conti, Ulric Ferner, Andrea Giorgetti, and Moe Z Win. Ranging with ultrawide bandwidth signals in multipath environments. Proceedings of the IEEE, 97(2):404-426, 2009.

[10] Krishna Chintalapudi, Anand Padmanabha Iyer, and Venkata N Padmanabhan. Indoor localization without the pain. In Proceedings of the sixteenth annual international conference on Mobile computing and networking, pages 173-184. ACM, 2010

[11] Moustafa Seifeldin, Ahmed Saeed, Ahmed E Kosba, Amr El-Keyi, and Moustafa Youssef. Nuzzer: A large-scale device-free passive localization system for wireless environments. Mobile Computing, IEEE Transactions on, 12(7):1321-1334, 2013.

[12] Anthea Wain Sy Au, Chen Feng, Shahrokh Valaee, Sophia Reyes, Sameh Sorour, Samuel N Markowitz, Deborah Gold, Kevaughn Gordon, and Moshe Eizenman. Indoor tracking and navigation using received signal strength and compressive sensing on a mobile device. Mobile Computing, IEEE Transactions on, 12(10):2050-2062, 2013.

[13] Pavel Davidson, Jussi Collin, and Jarmo Takala. Application of particle filters for indoor positioning using floor plans. In Ubiquitous Positioning Indoor Navigation and Location Based Service (UPINLBS), 2010, pages 1-4. IEEE, 2010.

[14] Begümhan Turgut and Richard P Martin. Restarting particle filters: an approach to improve the performance of dynamic indoor localization. In Global Telecommunications Conference, 2009. GLOBECOM 2009. IEEE, pages 1-7. IEEE, 2009.
[15] Henri Nurminen, Anssi Ristimaki, Simo Ali-Loytty, and Robert Piché. Particle filter and smoother for indoor localization. In Indoor Positioning and Indoor Navigation (IPIN), 2013 International Conference on, pages 1-10. IEEE, 2013.

[16] Henk Wymeersch, Stefano Maranò, Wesley M Gifford, and Moe Z Win. A machine learning approach to ranging error mitigation for uwb localization. Communications, IEEE Transactions on, 60(6):1719-1728, 2012.

[17] William PL Cully, Simon L Cotton, William G Scanlon, and JB McQuiston. Body shadowing mitigation using differentiated los/nlos channel models for rssi-based monte carlo personnel localization. In Wireless Communications and Networking Conference (WCNC), 2012 IEEE, pages 694-698. IEEE, 2012.

[18] Xinfeng Li, Jin Teng, Qiang Zhai, Junda Zhu, Dong Xuan, Yuan F Zheng, and Wei Zhao. Ev-human: Human localization via visual estimation of body electronic interference. In INFOCOM, 2013 Proceedings IEEE, pages 500-504. IEEE, 2013.

[19] Paolo Barsocchi, Francesco Furfari, Paolo Nepa, and Francesco Potortì. Rssi localisation with sensors placed on the user. In Indoor Positioning and Indoor Navigation (IPIN), 2010 International Conference on, pages 1-6. IEEE, 2010.

[20] Marco Altini, Davide Brunelli, Elisabetta Farella, and Luca Benini. Bluetooth indoor localization with multiple neural networks. In Wireless Pervasive Computing (ISWPC), 2010 5th IEEE International Symposium on, pages 295-300. IEEE, 2010.

[21] Zengbin Zhang, Xia Zhou, Weile Zhang, Yuanyang Zhang, Gang Wang, Ben Y Zhao, and Haitao Zheng. I am the antenna: accurate outdoor ap location using smartphones. In Proceedings of the 17th annual international conference on Mobile computing and networking, pages 109-120. ACM, 2011.

[22] Souvik Sen, Romit Roy Choudhury, and Srihari Nelakuditi. Spinloc: Spin once to know your location. In Proceedings of the Twelfth Workshop on Mobile Computing Systems \& Applications, page 12. ACM, 2012.

[23] Crossbow TelosB Datasheet. http://www.willow.co.uk/TelosB Datasheet.pdf. Accessed: 2015-06-16.

[24] Jens Trogh, David Plets, Luc Martens, and Wout Joseph. Advanced real-time indoor tracking based on the viterbi algorithm and semantic data. International Journal of Distributed Sensor Networks, 501:271818, 2015.

[25] David Plets, Wout Joseph, Kris Vanhecke, Emmeric Tanghe, and Luc Martens. Coverage prediction and optimization algorithms for indoor environments. EURASIP Journal on Wireless Communications and Networking, 2012(1):1-23, 2012.

[26] Andreas Christ, Wolfgang Kainz, Eckhart G Hahn, Katharina Honegger, Marcel Zefferer, Esra Neufeld, Wolfgang Rascher, Rolf Janka, Werner Bautz, Ji Chen, et al. The virtual familydevelopment of surfacebased anatomical models of two adults and two children for dosimetric simulations. Physics in medicine and biology, 55(2):N23, 2010.

[27] $2.4 \mathrm{GHz}$ Inverted $\mathrm{F}$ Antenna. http://www.ti.com/lit/an/swru120b/ swru120b.pdf. Accessed: 2015-06-16.

[28] Andreas F Molisch, Kannan Balakrishnan, Dajana Cassioli, Chia-Chin Chong, Shahriar Emami, Andrew Fort, Johan Karedal, Juergen Kunisch, Hans Schantz, Ulrich Schuster, et al. Ieee 802.15. 4a channel model-final report. IEEE P802, 15(04):0662, 2004.

[29] Moustafa Alzantot and Moustafa Youssef. Uptime: Ubiquitous pedestrian tracking using mobile phones. In Wireless Communications and Networking Conference (WCNC), 2012 IEEE, pages 3204-3209. IEEE, 2012. 